Research Article

\title{
Sharp Bounds for the Inverse Sum Indeg Index of Graph Operations
}

\author{
Anam Rani, ${ }^{1}$ Muhammad Imran $\mathbb{D}^{2},{ }^{2}$ and Usman Ali $\mathbb{D}^{3,4}$ \\ ${ }^{1}$ Department of Basic Sciences, Deanship of Preparatory Year, King Faisal University, Al Hofuf, Al Ahsa, Saudi Arabia \\ ${ }^{2}$ Department of Mathematical Sciences, United Arab Emirates University, P.O. Box 15551, Al Ain, UAE \\ ${ }^{3}$ Institute de Mathematiques de Jussieu-Paris Rive Gauche, (Universite de Paris/Sorbonne Universite), Paris, France \\ ${ }^{4}$ CASPAM, Bahauddin Zakariya University, Multan 66000, Pakistan
}

Correspondence should be addressed to Usman Ali; uali@bzu.edu.pk

Received 8 February 2021; Accepted 22 May 2021; Published 9 June 2021

Academic Editor: Toqeer Mahmood

Copyright $\odot 2021$ Anam Rani et al. This is an open access article distributed under the Creative Commons Attribution License, which permits unrestricted use, distribution, and reproduction in any medium, provided the original work is properly cited.

\begin{abstract}
Vukičević and Gasperov introduced the concept of 148 discrete Adriatic indices in 2010. These indices showed good predictive properties against the testing sets of the International Academy of Mathematical Chemistry. Among these indices, twenty indices were taken as beneficial predictors of physicochemical properties. The inverse sum indeg index denoted by $\operatorname{ISI}\left(\mathbf{G}_{k}\right)$ of $\mathbf{G}_{k}$ is a notable predictor of total surface area for octane isomers and is presented as ISI $\left(\mathbf{G}_{k}\right)=$ $\sum_{g_{k} g_{k}^{\prime} \in\left(\mathbf{G}_{k}\right)}\left(d_{\mathbf{G}_{k}}\left(g_{k}\right) d_{\mathbf{G}_{k}}\left(g_{k}^{\prime}\right) / d_{\mathbf{G}_{k}}\left(g_{k}\right)+d_{\mathbf{G}_{k}}\left(g_{k}^{\prime}\right)\right)$, where $d_{\mathbf{G}_{k}}\left(g_{k}\right)$ represents the degree of $g_{k} \in V\left(\mathbf{G}_{k}\right)$. In this paper, we determine sharp bounds for ISI index of graph operations, including the Cartesian product, tensor product, strong product, composition, disjunction, symmetric difference, corona product, Indu-Bala product, union of graphs, double graph, and strong double graph.
\end{abstract}

\section{Introduction}

Let $\mathbf{G}_{k}$ be a connected and simple graph whose vertex and edge sets are $V\left(\mathbf{G}_{k}\right)$ and $E\left(\mathbf{G}_{k}\right)$, respectively. The order $k$ and size $k^{\prime}$ of $\mathbf{G}_{k}$ are the cardinalities of $\left|V\left(\mathbf{G}_{k}\right)\right|$ and $\left|E\left(\mathbf{G}_{k}\right)\right|$, respectively. The degree formula of $g_{k} \in V\left(\mathbf{G}_{k}\right)$ is the cardinality of linked vertices to $g_{k}$ in $\mathbf{G}_{k}$ and represented by $d_{\mathbf{G}_{k}}\left(g_{k}\right)$. The largest (or smallest) degree of $\mathbf{G}_{k}$ is the degree of a vertex of $\mathbf{G}_{k}$ with the greatest (or least) number of edges incident to it and represented by $\Delta\left(\mathbf{G}_{k}\right)$ (or $\delta\left(\mathbf{G}_{k}\right)$ ).

A molecular descriptor is a numerical parameter of a graph that distinguished its topology. In organic chemistry, topological descriptors have investigated many applications in pharmaceutical drug design, QSAR/QSPR study, chemical documentation, and isomer discrimination. Some of these topological indices are Wiener index, Zagreb indices, Szeged index, and Randić index. The set of 148 discrete Adriatic descriptors [1] have been defined in 2010. These descriptors showed well predictive characteristics on the testing sets given by International Academy of Mathematical Chemistry. Twenty of these descriptors were taken as noteworthy predictors of physicochemical properties. One such index is inverse sum indeg index, denoted by $\operatorname{ISI}\left(\mathbf{G}_{k}\right)$, of $\mathbf{G}_{k}$ that was investigated in [1] as a noteworthy predictor of total surface area for octane isomers and is presented as follows:

$$
\operatorname{ISI}\left(\mathbf{G}_{k}\right)=\sum_{g_{k} g_{k}^{\prime} \in E\left(\mathbf{G}_{k}\right)} \frac{d_{\mathbf{G}_{k}}\left(g_{k}\right) d_{\mathbf{G}_{k}}\left(g_{k}^{\prime}\right)}{d_{\mathbf{G}_{k}}\left(g_{k}\right)+d_{\mathbf{G}_{k}}\left(g_{k}^{\prime}\right)} .
$$

Sedlar et al. [2] investigated graph-theoretical characteristics of ISI index. Falahati-Nezhad et al. [3] computed some sharp bounds of inverse sum indeg (ISI) index.

The Zagreb indices of $\mathbf{G}_{k}$ are presented by Gutman and Trinajstić [4] as follows:

$$
\begin{aligned}
& M_{1}\left(\mathbf{G}_{k}\right)=\sum_{g_{k} \in V\left(\mathbf{G}_{k}\right)} d_{\mathbf{G}_{k}}\left(g_{k}\right)^{2}, \\
& M_{2}\left(\mathbf{G}_{k}\right)=\sum_{g_{k} g_{k}^{\prime} \in E\left(\mathbf{G}_{k}\right)} d_{\mathbf{G}_{k}}\left(g_{k}\right) d_{\mathbf{G}_{k}}\left(g_{k}^{\prime}\right) .
\end{aligned}
$$


Let $\mathbf{G}_{k}$ be $k$-vertex and $\mathbf{H}_{l}$ be $l$-vertex graphs with size $k^{\prime}$ and $l^{\prime}$, respectively. The Cartesian product $\mathbf{G}_{k} \triangleright \mathbf{H}_{l}$, whose vertex set is $V\left(\mathbf{G}_{k}\right) \times V\left(\mathbf{H}_{l}\right)$ and $\left(g_{k}, h_{l}\right)$ and $\left(g_{k}^{\prime}, h_{l}^{\prime}\right)$ are adjacent when $g_{k}=g_{k}^{\prime}$ and $h_{l} h_{l}^{\prime} \in E\left(\mathbf{H}_{l}\right)$ or $g_{k} g_{k}^{\prime} \in E\left(\mathbf{G}_{k}\right)$ and $h_{l}=h_{l}^{\prime}$, is a graph. The order and size of $\mathbf{G}_{k} \triangleright \mathbf{H}_{l}$ are $k l$ and $k^{\prime} l+k l^{\prime}$, respectively. The degree formula for $\left(g_{k}, h_{l}\right) \in V\left(\mathbf{G}_{k} \triangleright \mathbf{H}_{l}\right)$ is $d_{\mathbf{G}_{k}}\left(g_{k}\right)+d_{\mathbf{H}_{l}}\left(h_{l}\right)$.

The tensor product $\mathbf{G}_{k} \times \mathbf{H}_{l}$, whose set of vertices is $V\left(\mathbf{G}_{k}\right) \times V\left(\mathbf{H}_{l}\right)$ and $\left(g_{k}, h_{l}\right)$ and $\left(g_{k}^{\prime}, h_{l}^{\prime}\right)$ are linked when $g_{k} g_{k}^{\prime} \in E\left(\mathbf{G}_{k}\right)$ and $h_{l} h_{l}^{\prime} \in E\left(\mathbf{H}_{l}\right)$, is a graph. The order and size of $\mathbf{G}_{k} \times \mathbf{H}_{l}$ are $k l$ and $2 k^{\prime} l^{\prime}$, respectively. The degree formula for $\left(g_{k}, h_{l}\right)$ in $\mathbf{G}_{k} \times \mathbf{H}_{l}$ is $d_{\mathbf{G}_{k}}\left(g_{k}\right) d_{\mathbf{H}_{l}}\left(h_{l}\right)$.

The strong product $\mathbf{G}_{k} \otimes \mathbf{H}_{l}$, whose vertex set and edge set are $V\left(\mathbf{G}_{k}\right) \times V\left(\mathbf{H}_{l}\right)$ and $E\left(\mathbf{G}_{k} \triangleright \mathbf{H}_{l}\right) \cup E\left(\mathbf{G}_{k} \times \mathbf{H}_{l}\right)$, respectively, is a graph. The order and size of $\mathbf{G}_{k} \otimes \mathbf{H}_{l}$ are $k l$ and $k l^{\prime}+l k^{\prime}+2 k^{\prime} l^{\prime}$, respectively. The degree formula for $\left(g_{k}, h_{l}\right)$ in $\mathbf{G}_{k} \otimes \mathbf{H}_{l}$ is $d_{\mathbf{G}_{k}}\left(g_{k}\right)+d_{\mathbf{H}_{l}}\left(h_{l}\right)+d_{\mathbf{G}_{k}}\left(g_{k}\right) d_{\mathbf{H}_{l}}\left(h_{l}\right)$.

The composition $\mathbf{G}_{k}\left[\mathbf{H}_{l}\right]$, whose vertex set $V\left(\mathbf{G}_{k}\right) \times V\left(\mathbf{H}_{l}\right)$ and $\left(g_{k}, h_{l}\right)$ and $\left(g_{k}^{\prime}, h_{l}^{\prime}\right)$ are linked when $g_{k} g_{k}^{\prime} \in E\left(\mathbf{G}_{k}\right)$ or $g_{k}=g_{k}^{\prime}$ and $h_{l} h_{l}^{\prime} \in E\left(\mathbf{H}_{l}\right)$, is a graph. The order and size of $\mathbf{G}_{k}\left[\mathbf{H}_{l}\right]$ are $k l$ and $k^{\prime} l^{2}+k l^{\prime}$, respectively. The degree formula for $\left(g_{k}, h_{l}\right)$ in $\mathbf{G}_{k}\left[\mathbf{H}_{l}\right]$ is $l d_{\mathbf{G}_{k}}\left(g_{k}\right)$ $+d_{\mathbf{H}_{l}}\left(h_{l}\right)$.

The disjunction $\mathbf{G}_{k} \vee \mathbf{H}_{l}$, whose vertex set is $V\left(\mathbf{G}_{k}\right) \times$ $V\left(\mathbf{H}_{l}\right)$ and $\left(g_{k}, h_{l}\right)$ and $\left(g_{k}^{\prime}, h_{l}^{\prime}\right)$ are linked when $g_{k} g_{k}^{\prime} \in E\left(\mathbf{G}_{k}\right)$ or $h_{l} h_{\epsilon}^{\prime} E\left(\mathbf{H}_{l}\right)$ is a graph. The order and size of $\mathbf{G}_{k} \vee \mathbf{H}_{l}$ are $k l$ and $k l^{2}+l ı k^{2}-2 k \prime l ı$, respectively. The degree formula for $\left(g_{k}, h_{l}\right)$ in $\mathbf{G}_{k} \vee \mathbf{H}_{l}$ is $l d_{\mathbf{G}_{k}}\left(g_{k}\right)+k d_{\mathbf{H}_{l}}\left(h_{l}\right)-$ $d_{\mathbf{G}_{k}}\left(g_{k}\right) d_{\mathbf{H}_{l}}\left(h_{l}\right)$.

The symmetric difference $\mathbf{G}_{k} \oplus \mathbf{H}_{l}$ is a graph with vertex set $V\left(\mathbf{G}_{k}\right) \times V\left(\mathbf{H}_{l}\right)$ and $\left(g_{k}, h_{l}\right)\left(g_{k}^{\prime}, h_{l}^{\prime}\right) \in E\left(\mathbf{G}_{k} \oplus \mathbf{H}_{l}\right)$ whenever $\left[g_{k} g_{k}^{\prime} \in E\left(\mathbf{G}_{k}\right)\right]$ or $\left[h_{l} h_{l}^{\prime} \in E\left(\mathbf{H}_{l}\right)\right]$ but not both. The order and size of $\mathbf{G}_{k} \oplus \mathbf{H}_{l}$ are $k l$ and $k \prime l^{2}+l^{\prime} k^{2}-4 k l$, respectively. The degree formula for $\left(g_{k}, h_{l}\right) \in V\left(\mathbf{G}_{k} \oplus \mathbf{H}_{l}\right)$ is $l d_{\mathbf{G}_{k}}\left(g_{k}\right)+k d_{\mathbf{H}_{l}}\left(h_{l}\right)-2 d_{\mathbf{G}_{k}}\left(g_{k}\right) d_{\mathbf{H}_{l}}\left(h_{l}\right)$.

Let $\mathbf{G}_{k_{1}}, \mathbf{G}_{k_{2}}, \ldots, \mathbf{G}_{k_{n}}$ be all vertex disjoint graphs. Then, their join is a graph whose vertex set is $\cup_{s=1}^{n} V\left(\mathbf{G}_{k_{s}}\right)$ and edge set is $\cup_{s=1}^{n} E\left(\mathbf{G}_{k_{s}}\right)$ together with the edges linking $V\left(\mathbf{G}_{k_{1}}\right)$ and $V\left(\mathbf{G}_{k_{2}}\right), V\left(\mathbf{G}_{k_{2}}\right)$ and $V\left(\mathbf{G}_{k_{3}}\right)$ so on $V\left(\mathbf{G}_{k_{n-1}}\right)$ and $V\left(\mathbf{G}_{k_{n}}\right)$. The degree formula of $g_{k} \in V\left(\mathbf{G}_{k_{1}}+\mathbf{G}_{k_{2}}+\cdots+\mathbf{G}_{k_{n}}^{n}\right)$ is $d_{\mathbf{G}_{k_{s}}}\left(g_{k}\right)+r-k_{s}, s=1,2, \ldots, n$ and $r=k_{1}+k_{2}+\cdots+k_{n}$.

The corona product $\mathbf{G}_{k} \circ \mathbf{H}_{l}$ is acquired by taking $\mathbf{G}_{k}$ as a single copy and $k$ copies of $\mathbf{H}_{l}$ and by linking $r$-th vertex of $\mathbf{G}_{k}$ to every vertex of $r$-th copy of $\mathbf{H}_{l}$, where $1 \leq r \leq k$. The graph $\mathbf{G}_{k} \circ \mathbf{H}_{l}$ has size and order $k^{\prime}+k l^{\prime}+k l$ and $k(1+l)$, respectively. The degree formula of $g \in V\left(\mathbf{G}_{k} \circ \mathbf{H}_{l}\right)$ is

$$
d_{\mathbf{G}_{k} \circ \mathbf{H}_{l}}(g)= \begin{cases}d_{\mathbf{G}_{k}}(g)+l, & \text { for } g \in V\left(\mathbf{G}_{k}\right), \\ d_{\mathbf{H}_{l}}(g)+1, & \text { for } g \in V\left(\mathbf{H}_{l}\right) .\end{cases}
$$

The Indu-Bala product $\mathbf{G}_{k} \boldsymbol{\nabla} \mathbf{H}_{l}$ is obtained from two disjoint copies of $\mathbf{G}_{k}+\mathbf{H}_{l}$ by linking the corresponding vertices of two copies of $\mathbf{H}_{l}$. The order and size of $\mathbf{G}_{k} \boldsymbol{\nabla} \mathbf{H}_{l}$ are $2(k+l)$ and $2 k^{\prime}+2 l^{\prime}+2 k l+l$, respectively. The degree of $g \in V\left(\mathbf{G}_{k} \boldsymbol{\nabla} \mathbf{H}_{l}\right)$ is

$$
d_{\mathbf{G}_{k} \mathbf{\nabla} \mathbf{H}_{l}}(g)= \begin{cases}d_{\mathbf{G}_{k}}(g)+l, & \text { for } g \in V\left(\mathbf{G}_{k}\right), \\ d_{\mathbf{H}_{l}}(g)+k+1, & \text { for } g \in V\left(\mathbf{H}_{l}\right) .\end{cases}
$$

The double graph $D\left[\mathbf{G}_{k}\right]$ is acquired by taking original edge set of two copies $V_{1}\left(\mathbf{G}_{k}\right)$ and $V_{2}\left(\mathbf{G}_{k}\right)$ of $V\left(\mathbf{G}_{k}\right)$ and linking each vertex in $V_{1}\left(\mathbf{G}_{k}\right)$ with the linked vertices of corresponding vertex in $V_{2}\left(\mathbf{G}_{k}\right)$. The strong double graph $\mathrm{SD}\left[\mathbf{G}_{k}\right]$ is acquired by taking two copies of $V_{1}\left(\mathbf{G}_{k}\right)$ and $V_{2}\left(\mathbf{G}_{k}\right)$ of $V\left(\mathbf{G}_{k}\right)$ and linking each vertex in $V_{1}\left(\mathbf{G}_{k}\right)$ with closed neighborhood of corresponding vertex in $V_{2}\left(\mathbf{G}_{k}\right)$.

Figure 1 depicts some graph operations. For more details on these graph operations, see [5-14]. Also, we refer some recent articles [15-19] on different kinds of descriptors.It is an important and well-reputed problem to study and explore the molecular topological descriptors of the graph operations in terms of the original graphs, say $\mathbf{G}_{k}$ and $\mathbf{H}_{l}$, and this also helps to explore the physicochemical properties of the complex chemical structures which arise from these graph operations. The upper and lower bounds of any molecular descriptors are the important information related to a chemical graph. They determine the approximate possible range of the invariant in the form of molecular structural parameters. There are some bounds already available for the inverse sum indeg (ISI) index regarding the number of pendant vertices, size, radius, smallest and largest vertex degrees, and smallest nonpendent vertex degree of a graph computed in [3]. The objective of this article is to determine the bounds for inverse sum indeg index of some graph operations including Cartesian product, tensor product, strong product, composition, disjunction, symmetric difference, corona product, Indu-Bala product, union of graphs, double graph, and strong double graph in the form of original graphs, say $\mathbf{G}_{k}$ and $\mathbf{H}_{l}$.

\section{Applications of Graph Theory Concept and Topological Indices in Chemistry}

In 1936, Hosoya introduced the concept of graph terminologies in chemistry and provided a modeling for molecules. This modeling contents lead to predict the chemical properties of molecules, easy classification of chemical compounds, computer simulations, and computer-assisted design of new chemical compounds. As in current century, chemists manipulate graphs on a daily basis using Table 1 terminologies for recent development in their research.

Graph hypothesis had investigated an interesting exercise around in research. Compound graph speculation has provided a collection of beneficial indices, for instance, topological indices. The Zagreb indices are the topological indices that are correlated to a substantial computation of fabricated characteristics of the particles and have been investigated parallel to establishing the Kovats constants and limit of the particles [20]. The hyper Zagreb descriptor has a strong bound between the security of direct dendrimers besides the expanded medication stores and for establishing the strain criticalness of cyclo alkanes [21]. To connect with various physico-mix characteristics, Zagreb indices have required deep control upon the essentialness of the dendrimers [22]. The Zagreb polynomials were determined to happen for computation of the $\pi$-electron imperativeness of the particles inside specific brutal verbalizations $[23,24]$. 


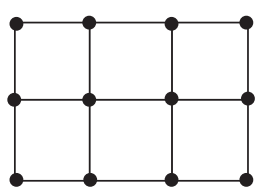

(a)

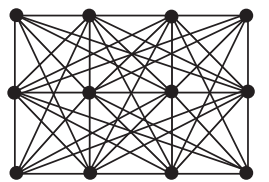

(f)

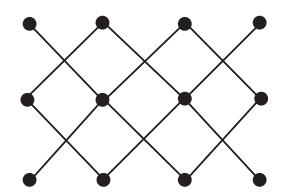

(b)

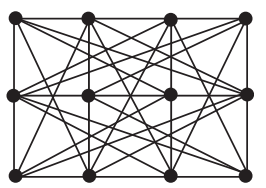

(g)

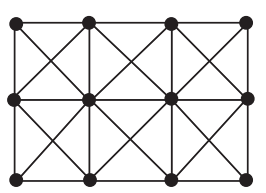

(c)

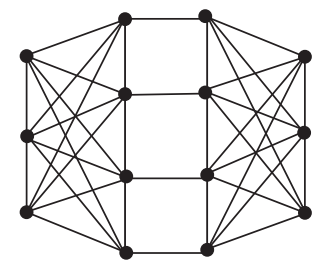

(h)

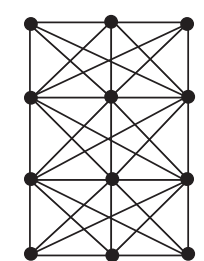

(d)

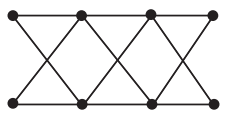

(i)

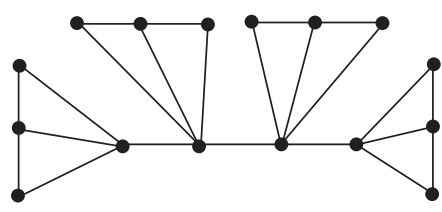

(e)

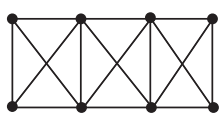

(j)

Figure 1: Graph operations: (a) $P_{3} \square P_{4}$; (b) $P_{3} \times P_{4}$; (c) $P_{3} \otimes P_{4}$; (d) $P_{4}\left[P_{3}\right]$; (e) $P_{4} \circ P_{3}$; (f) $P_{3} \vee P_{4}$; (g) $P_{3} \oplus P_{4}$; (h) $P_{3} \nabla P_{4}$; (i) $D\left[P_{4}\right]$; (j) $\operatorname{SD}\left[P_{4}\right]$.

TABLE 1: Graph theory and chemistry dictionary.

Graph theory

Graph

Vertex

Edge

Vertex degree

Tree

Bipartite graph

Perfect matching

Adjacency matrix

Chemistry

Structural formula

$$
\text { Atom }
$$

Chemical bond

Valency of atom

Acyclic structure

Alternant structure

Kekule structure

Huckel matrix

\section{Inverse Sum Indeg Index of Graph Operations}

In this section, we compute the inverse sum indeg index of the Cartesian product, tensor product, strong product, composition, disjunction, symmetric difference, corona product, Indu-Bala product, double graph, and strong double graph. The relation between largest and smallest degree of $\mathbf{G}_{k}$ to the degree of $g_{k} \in V\left(\mathbf{G}_{k}\right)$ is as follows:

$$
\begin{aligned}
& d_{\mathbf{G}_{k}}\left(g_{k}\right) \leq \Delta_{\mathbf{G}_{k}}, \\
& d_{\mathbf{G}_{k}}\left(g_{k}\right) \geq \delta_{\mathbf{G}_{k}} .
\end{aligned}
$$

In the upcoming theorem, we calculate the bounds for inverse sum indeg (ISI) index of Cartesian product.

Theorem 1. Let $\mathbf{G}_{k}$ and $\mathbf{H}_{l}$ be two graphs. Then,

$$
\frac{M_{2}\left(\mathbf{G}_{k} \triangleright \mathbf{H}_{l}\right)}{2\left(\Delta_{\mathbf{G}_{k}}+\Delta_{\mathbf{H}_{l}}\right)} \leq \operatorname{ISI}\left(\mathbf{G}_{k} \triangleright \mathbf{H}_{l}\right) \leq \frac{M_{2}\left(\mathbf{G}_{k} \triangleright \mathbf{H}_{l}\right)}{2\left(\delta_{\mathbf{G}_{k}}+\delta_{\mathbf{H}_{l}}\right)} \text {. }
$$

The equalities hold if and only if $\mathbf{G}_{k}$ and $\mathbf{H}_{l}$ are regular.

Proof. Using the degree formula for a vertex of $\mathbf{G}_{k} \triangleright \mathbf{H}_{l}$ in equation (1),

$$
\begin{aligned}
\operatorname{ISI}\left(\mathbf{G}_{k} \triangleright \mathbf{H}_{l}\right) & =\sum_{\left(g_{k}, h_{l}\right)\left(g_{k}^{\prime} h_{l}^{\prime}\right) \in E\left(\mathbf{G}_{k} \triangleright \mathbf{H}_{l}\right)} \frac{d_{\mathbf{G}_{k} \triangleright \mathbf{H}_{l}}\left(g_{k}, h_{l}\right) d_{\mathbf{G}_{k} \triangleright \mathbf{H}_{l}}\left(g_{k}^{\prime}, h_{l}^{\prime}\right)}{d_{\mathbf{G}_{k} \triangleright \mathbf{H}_{l}}\left(g_{k}, h_{l}\right)+d_{\mathbf{G}_{k} \triangleright \mathbf{H}_{l}}\left(g_{k}^{\prime}, h_{l}^{\prime}\right)} \\
& =\sum_{\left(g_{k}, h_{l}\right)\left(g_{k}^{\prime} h_{l}^{\prime}\right) \in E\left(\mathbf{G}_{k} \triangleright \mathbf{H}_{l}\right)} \frac{d_{\mathbf{G}_{k} \triangleright \mathbf{H}_{l}}\left(g_{k}, h_{l}\right) d_{\mathbf{G}_{k} \triangleright \mathbf{H}_{l}}\left(g_{k}^{\prime}, h_{l}^{\prime}\right)}{d_{\mathbf{G}_{k}}\left(g_{k}\right)+d_{\mathbf{H}_{l}}\left(h_{l}\right)+d_{\mathbf{G}_{k}}\left(g_{k}^{\prime}\right)+d_{\mathbf{H}_{l}}\left(h_{l}^{\prime}\right)} \\
& \leq \frac{1}{2\left(\delta_{\mathbf{G}_{k}}+\delta_{\left.\mathbf{H}_{l}\right)}\right.}\left(\sum_{k}, h_{l}\right) \\
& =\frac{M_{2}\left(\mathbf{G}_{k} \triangleright \mathbf{H}_{l}^{\prime}\right) \in E\left(\mathbf{G}_{k} \triangleright \mathbf{H}_{l}\right)}{2\left(\delta_{\mathbf{G}_{k}}+\delta_{\left.\mathbf{H}_{l}\right)}\right)} .
\end{aligned}
$$

Similarly, we can evaluate

$$
\operatorname{ISI}\left(\mathbf{G}_{k} \triangleright \mathbf{H}_{l}\right) \geq \frac{M_{2}\left(\mathbf{G}_{k} \triangleright \mathbf{H}_{l}\right)}{2\left(\Delta_{\mathbf{G}_{k}}+\Delta_{\mathbf{H}_{l}}\right)} \text {. }
$$

The above equalities hold if and only if factor graphs are regular.

In the next theorem, we calculate the bounds for ISI index of tensor product of $\mathbf{G}_{k}$ and $\mathbf{H}_{l}$. 
Theorem 2. Let $\mathbf{G}_{k}$ and $\mathbf{H}_{l}$ be two graphs. Then,

$$
\frac{M_{2}\left(\mathbf{G}_{k}\right) M_{2}\left(\mathbf{H}_{l}\right)}{\Delta_{\mathbf{G}_{k}} \Delta_{\mathbf{H}_{l}}} \leq \operatorname{ISI}\left(\mathbf{G}_{k} \times \mathbf{H}_{l}\right) \leq \frac{M_{2}\left(\mathbf{G}_{k}\right) M_{2}\left(\mathbf{H}_{l}\right)}{\delta_{\mathbf{G}_{k}} \delta_{\mathbf{H}_{l}}} .
$$

The above equalities hold if and only if both graphs are regular.

Proof. Using the degree formula for a vertex in tensor product of graphs in (1),

$$
\begin{aligned}
& \operatorname{ISI}\left(\mathbf{G}_{k} \times \mathbf{H}_{l}\right)= \sum_{\left(g_{k}, h_{l}\right)\left(g_{k}^{\prime} h_{l}^{\prime}\right) \in E\left(\mathbf{G}_{k} \times \mathbf{H}_{l}\right)} \frac{d_{\mathbf{G}_{k} \times \mathbf{H}_{l}}\left(g_{k}, h_{l}\right) d_{\mathbf{G}_{k} \times \mathbf{H}_{l}}\left(g_{k}^{\prime}, h_{l}^{\prime}\right)}{d_{\mathbf{G}_{k} \times \mathbf{H}_{l}}\left(g_{k}, h_{l}\right)+d_{\mathbf{G}_{k} \times \mathbf{H}_{l}}\left(g_{k}^{\prime}, h_{l}^{\prime}\right)} \\
&= \sum_{\left(g_{k}, h_{l}\right)\left(g_{k}^{\prime} h_{l}^{\prime}\right) \in E\left(\mathbf{G}_{k} \times \mathbf{H}_{l}\right)} \frac{d_{\mathbf{G}_{k} \times \mathbf{H}_{l}}\left(g_{k}, h_{l}\right) d_{\mathbf{G}_{k} \times \mathbf{H}_{l}}\left(g_{k}^{\prime}, h_{l}^{\prime}\right)}{d_{\mathbf{G}_{k}}\left(g_{k}\right) d_{\mathbf{H}_{l}}\left(h_{l}\right)+d_{\mathbf{G}_{k}}\left(g_{k}^{\prime}\right) d_{\mathbf{H}_{l}}\left(h_{l}^{\prime}\right)} \\
& \leq \frac{1}{2 \delta_{\mathbf{G}_{k}} \delta_{\mathbf{H}_{l}}}\left(g_{k}, h_{l}\right)\left(g_{k}^{\prime} h_{l}^{\prime}\right) \in E\left(\mathbf{G}_{k} \times \mathbf{H}_{l}\right) \\
& d_{\mathbf{G}_{k} \times \mathbf{H}_{l}}\left(g_{k}, h_{l}\right) d_{\mathbf{G}_{k} \times \mathbf{H}_{l}}\left(g_{k}^{\prime}, h_{l}^{\prime}\right) \\
&= \\
&=\frac{M_{2}\left(\mathbf{G}_{k} \times \mathbf{H}_{l}\right)}{2 \delta_{\mathbf{G}_{k}} \delta_{\mathbf{H}_{l}}} \\
&=\frac{M_{2}\left(\mathbf{G}_{k}\right) M_{2}\left(\mathbf{H}_{l}\right)}{\delta_{\mathbf{G}_{k}} \delta_{\mathbf{H}_{l}}} .
\end{aligned}
$$

See Theorem 2.1 in [25]. Similarly, we can compute

$$
\operatorname{ISI}\left(\mathbf{G}_{k} \times \mathbf{H}_{l}\right) \geq \frac{M_{2}\left(\mathbf{G}_{k}\right) M_{2}\left(\mathbf{H}_{l}\right)}{\Delta_{\mathbf{G}_{k}} \Delta_{\mathbf{H}_{l}}} .
$$

The above equalities hold if and only if factor graphs are regular.

We derive the bounds of inverse sum indeg (ISI) index of $\mathbf{G}_{k} \otimes \mathbf{H}_{l}$ in the upcoming theorem.

Theorem 3. Let $\mathbf{G}_{k}$ and $\mathbf{H}_{l}$ be two graphs. Then,

$$
\frac{M_{2}\left(\mathbf{G}_{k} \otimes \mathbf{H}_{l}\right)}{2\left(\Delta_{\mathbf{G}_{k}}+\Delta_{\mathbf{H}_{l}}+\Delta_{\mathbf{G}_{k}} \Delta_{\mathbf{H}_{l}}\right)} \leq \operatorname{ISI}\left(\mathbf{G}_{k} \otimes \mathbf{H}_{l}\right) \leq \frac{M_{2}\left(\mathbf{G}_{k} \otimes \mathbf{H}_{l}\right)}{2\left(\delta_{\mathbf{G}_{k}}+\delta_{\mathbf{H}_{l}}+\delta_{\mathbf{G}_{k}} \delta_{\mathbf{H}_{l}}\right)} .
$$

The equalities hold if and only if both graphs are regular.

Proof. Using the degree formula of a vertex in strong product of graphs in (1),

$$
\begin{aligned}
\operatorname{ISI}\left(\mathbf{G}_{k} \times \mathbf{H}_{l}\right)= & \sum_{\left(g_{k}, h_{l}\right)\left(g_{k}^{\prime} h_{l}^{\prime}\right) \in E\left(\mathbf{G}_{k} \otimes \mathbf{H}_{l}\right)} \frac{d_{\mathbf{G}_{k} \otimes \mathbf{H}_{l}}\left(g_{k}, h_{l}\right) d_{\mathbf{G}_{k} \otimes \mathbf{H}_{l}}\left(g_{k}^{\prime}, h_{l}^{\prime}\right)}{d_{\mathbf{G}_{k} \otimes \mathbf{H}_{l}}\left(g_{k}, h_{l}\right)+d_{\mathbf{G}_{k} \otimes \mathbf{H}_{l}}\left(g_{k}^{\prime}, h_{l}^{\prime}\right)} \\
= & \sum_{\left(g_{k}, h_{l}\right)\left(g_{k}^{\prime} h_{l}^{\prime}\right) \in E\left(\mathbf{G}_{k} \triangleright \mathbf{H}_{l}\right)} \frac{d_{\mathbf{G}_{k} \otimes \mathbf{H}_{l}}\left(g_{k}, h_{l}\right) d_{\mathbf{G}_{k} \otimes \mathbf{H}_{l}}\left(g_{k}^{\prime}, h_{l}^{\prime}\right)}{d_{\mathbf{G}_{k}}\left(g_{k}\right)+d_{\mathbf{H}_{l}}\left(h_{l}\right)+d_{\mathbf{G}_{k}}\left(g_{k}\right) d_{\mathbf{H}_{l}}\left(h_{l}\right)+d_{\mathbf{G}_{k}}\left(g_{k}^{\prime}\right) d_{\mathbf{H}_{l}}\left(h_{l}^{\prime}\right)+d_{\mathbf{G}_{k}}\left(g_{k}\right)+d_{\mathbf{H}_{l}}\left(h_{l}^{\prime}\right)} \\
\leq & \frac{1}{2\left(\delta_{\mathbf{G}_{k}}+\delta_{\mathbf{H}_{l}}+\delta_{\mathbf{G}_{k}} \delta_{\left.\mathbf{H}_{l}\right)}\right)} \sum_{\left(g_{k}, h_{l}\right)\left(g_{k}^{\prime} h_{l}^{\prime}\right) \in E\left(\mathbf{G}_{k} \triangleright \mathbf{H}_{l}\right)} d_{\mathbf{G}_{k} \otimes \mathbf{H}_{l}}\left(g_{k}, h_{l}\right) d_{\mathbf{G}_{k} \otimes \mathbf{H}_{l}}\left(g_{k}^{\prime}, h_{l}^{\prime}\right) \\
& =\frac{M_{2}\left(\mathbf{G}_{k} \times \mathbf{H}_{l}\right)}{2\left(\delta_{\mathbf{G}_{k}}+\delta_{\mathbf{H}_{l}}+\delta_{\mathbf{G}_{k}} \delta_{\mathbf{H}_{l}}\right)} .
\end{aligned}
$$

In a similarly way, 


$$
\operatorname{ISI}\left(\mathbf{G}_{k} \otimes \mathbf{H}_{l}\right) \geq \frac{M_{2}\left(\mathbf{G}_{k} \otimes \mathbf{H}_{l}\right)}{2\left(\Delta_{\mathbf{G}_{k}}+\Delta_{\mathbf{H}_{l}}+\Delta_{\mathbf{G}_{k}} \Delta_{\mathbf{H}_{l}}\right)} .
$$

The above equalities satisfy if and only if factor graphs are regular.

In the upcoming theorem, we evaluate the bounds for inverse sum indeg (ISI) index of $\mathbf{G}_{k}\left[\mathbf{H}_{l}\right]$.

Theorem 4. Let $\mathbf{G}_{k}$ and $\mathbf{H}_{l}$ be two graphs. Then,

$$
\frac{M_{2}\left(\mathbf{G}_{k}\left[\mathbf{H}_{l}\right]\right)}{2\left(l \Delta_{\mathbf{G}_{k}}+\Delta_{\mathbf{H}_{l}}\right)} \leq \operatorname{ISI}\left(\mathbf{G}_{k}\left[\mathbf{H}_{l}\right]\right) \leq \frac{M_{2}\left(\mathbf{G}_{k}\left[\mathbf{H}_{l}\right]\right)}{2\left(l \delta_{\mathbf{G}_{k}}+\delta_{\mathbf{H}_{l}}\right)} .
$$

The equalities hold if and only if both graphs are regular.

Proof. Using the degree formula of an element of $V\left(\mathbf{G}_{k}\left[\mathbf{H}_{l}\right]\right)$ in (1),

$$
\begin{aligned}
\operatorname{ISI}\left(\mathbf{G}_{k}\left[\mathbf{H}_{l}\right]\right)= & \sum_{\left(g_{k}, h_{l}\right)\left(g_{k}^{\prime} h_{l}^{\prime}\right) \in E\left(\mathbf{G}_{k}\left[\mathbf{H}_{l}\right]\right)} \frac{d_{\mathbf{G}_{k}\left[\mathbf{H}_{l}\right]}\left(g_{k}, h_{l}\right) d_{\mathbf{G}_{k}\left[\mathbf{H}_{l}\right]}\left(g_{k}^{\prime}, h_{l}^{\prime}\right)}{d_{\mathbf{G}_{k}\left[\mathbf{H}_{l}\right]}\left(g_{k}, h_{l}\right)+d_{\mathbf{G}_{k}\left[\mathbf{H}_{l}\right]}\left(g_{k}^{\prime}, h_{l}^{\prime}\right)} \\
= & \sum_{\left(g_{k}, h_{l}\right)\left(g_{k}^{\prime} h_{l}^{\prime}\right) \in E\left(\mathbf{G}_{k}\left[\mathbf{H}_{l}\right]\right)} \frac{d_{\mathbf{G}_{k}\left[\mathbf{H}_{l}\right]}\left(g_{k}, h_{l}\right) d_{\mathbf{G}_{k}\left[\mathbf{H}_{l}\right]}\left(g_{k}^{\prime}, h_{l}^{\prime}\right)}{l d_{\mathbf{G}_{k}}\left(g_{k}\right)+d_{\mathbf{H}_{l}}\left(h_{l}\right)+l d_{\mathbf{G}_{k}}\left(g_{k}^{\prime}\right)+d_{\mathbf{H}_{l}}\left(h_{l}^{\prime}\right)} \\
\leq & \frac{1}{2\left(l \delta_{\mathbf{G}_{k}}+\delta_{\left.\mathbf{H}_{l}\right)}\right)}\left(g_{k}, h_{l}\right)\left(g_{k}^{\prime} h_{l}^{\prime}\right) \in E\left(\mathbf{G}_{k}\left[\mathbf{H}_{l}\right]\right) \\
& d_{\mathbf{G}_{k}\left[\mathbf{H}_{l}\right]}\left(g_{k}, h_{l}\right) d_{\mathbf{G}_{k}\left[\mathbf{H}_{l}\right]}\left(g_{k}^{\prime}, h_{l}^{\prime}\right) \\
& =\frac{M_{2}\left(\mathbf{G}_{k}\left[\mathbf{H}_{l}\right]\right)}{2\left(l \delta_{\mathbf{G}_{k}}+\delta_{\left.\mathbf{H}_{l}\right)}\right)} .
\end{aligned}
$$

In a similar way,

$$
\operatorname{ISI}\left(\mathbf{G}_{k}\left[\mathbf{H}_{l}\right]\right) \geq \frac{M_{2}\left(\mathbf{G}_{k}\left[\mathbf{H}_{l}\right]\right)}{2\left(l \Delta_{\mathbf{G}_{k}}+\Delta_{\mathbf{H}_{l}}\right)}
$$

The above equalities hold if and only if factor graphs are regular.

In the following theorem, we present the bounds for inverse sum indeg (ISI) index of disjunction of $\mathbf{G}_{k}$ and $\mathrm{H}_{l}$.

$$
\begin{aligned}
\frac{M_{2}\left(\mathbf{G}_{k} \vee \mathbf{H}_{l}\right)}{2\left(l \Delta_{\mathbf{G}_{k}}+k \Delta_{\mathbf{H}_{l}}-\Delta_{\mathbf{G}_{k}} \Delta_{\mathbf{H}_{l}}\right)} & \leq \operatorname{ISI}\left(\mathbf{G}_{k} \vee \mathbf{H}_{l}\right) \\
& \leq \frac{M_{2}\left(\mathbf{G}_{k} \vee \mathbf{H}_{l}\right)}{2\left(l \delta_{\mathbf{G}_{k}}+k \delta_{\mathbf{H}_{l}}-\delta_{\mathbf{G}_{k}} \delta_{\mathbf{H}_{l}}\right)} .
\end{aligned}
$$

The equalities hold when factor graphs are regular.

Proof. Using the degree formula of an element of $V\left(\mathbf{G}_{k} \vee \mathbf{H}_{l}\right)$ in (1),

Theorem 5. Let $\mathbf{G}_{k}$ and $\mathbf{H}_{l}$ be two graphs. Then,

$$
\begin{aligned}
\operatorname{ISI}\left(\mathbf{G}_{k} \vee \mathbf{H}_{l}\right) & =\sum_{\left(g_{k}, h_{l}\right)\left(g_{k}^{\prime} h_{l}^{\prime}\right) \in E\left(\mathbf{G}_{k} \vee \mathbf{H}_{l}\right)} \frac{d_{\mathbf{G}_{k} \vee \mathbf{H}_{l}}\left(g_{k}, h_{l}\right) d_{\mathbf{G}_{k} \vee \mathbf{H}_{l}}\left(g_{k}^{\prime}, h_{l}^{\prime}\right)}{d_{\mathbf{G}_{k} \vee \mathbf{H}_{l}}\left(g_{k}, h_{l}\right)+d_{\mathbf{G}_{k} \vee \mathbf{H}_{l}}\left(g_{k}^{\prime}, h_{l}^{\prime}\right)} \\
& =\sum_{\left(g_{k}, h_{l}\right)\left(g_{k}^{\prime} h_{l}^{\prime}\right) \in E\left(\mathbf{G}_{k} \vee \mathbf{H}_{l}\right)} \frac{d_{\mathbf{G}_{k} \vee \mathbf{H}_{l}}\left(g_{k}, h_{l}\right) d_{\mathbf{G}_{k} \vee \mathbf{H}_{l}}\left(g_{k}^{\prime}, h_{l}^{\prime}\right)}{l d_{\mathbf{G}_{k}}\left(g_{k}\right)+k d_{\mathbf{H}_{l}}\left(h_{l}\right)-d_{\mathbf{G}_{k}}\left(g_{k}\right) d_{\mathbf{H}_{l}}\left(h_{l}\right)+l d_{\mathbf{G}_{k}}\left(g_{k}^{\prime}\right)+k d_{\mathbf{H}_{l}}\left(h_{l}^{\prime}\right)-d_{\mathbf{G}_{k}}\left(g_{k}^{\prime}\right) d_{\mathbf{H}_{l}}\left(h_{l}^{\prime}\right)} \\
& \leq \frac{1}{2\left(l \delta_{\mathbf{G}_{k}}+k \delta_{\mathbf{H}_{l}}-\delta_{\mathbf{G}_{k}} \delta_{\mathbf{H}_{l}}\right)} \\
& \sum_{\left(g_{k}, h_{l}\right)\left(g_{k}^{\prime} h_{l}^{\prime}\right) \in E\left(\mathbf{G}_{k} \vee \mathbf{H}_{l}\right)} d_{\mathbf{G}_{k} \vee \mathbf{H}_{l}}\left(g_{k}, h_{l}\right) d_{\mathbf{G}_{k} \vee \mathbf{H}_{l}}\left(g_{k}^{\prime}, h_{l}^{\prime}\right) \\
& =\frac{M_{2}\left(\mathbf{G}_{k} \vee \mathbf{H}_{l}\right)}{2\left(l \delta_{\mathbf{G}_{k}}+k \delta_{\mathbf{H}_{l}}-\delta_{\mathbf{G}_{k}} \delta_{\mathbf{H}_{l}}\right)} .
\end{aligned}
$$


Similarly, we compute

$$
\operatorname{ISI}\left(\mathbf{G}_{k} \vee \mathbf{H}_{l}\right) \geq \frac{M_{2}\left(\mathbf{G}_{k} \vee \mathbf{H}_{l}\right)}{2\left(l \Delta_{\mathbf{G}_{k}}+k \Delta_{\mathbf{H}_{l}}-\Delta_{\mathbf{G}_{k}} \Delta_{\mathbf{H}_{l}}\right)} .
$$

The above equalities hold when both graphs are regular.

Next, we derive the bounds of inverse sum indeg (ISI) index of $\mathbf{G}_{k} \oplus \mathbf{H}_{l}$.

Theorem 6. Let $\mathbf{G}_{k}$ and $\mathbf{H}_{l}$ be two graphs. Then,

$$
\begin{aligned}
\frac{M_{2}\left(\mathbf{G}_{k} \oplus \mathbf{H}_{l}\right)}{2\left(l \Delta_{\mathbf{G}_{k}}+k \Delta_{\mathbf{H}_{l}}-2 \Delta_{\mathbf{G}_{k}} \Delta_{\mathbf{H}_{l}}\right)} & \leq \operatorname{ISI}\left(\mathbf{G}_{k} \oplus \mathbf{H}_{l}\right) \\
& \leq \frac{M_{2}\left(\mathbf{G}_{k} \oplus \mathbf{H}_{l}\right)}{2\left(l \delta_{\mathbf{G}_{k}}+k \delta_{\mathbf{H}_{l}}-2 \delta_{\mathbf{G}_{k}} \delta_{\mathbf{H}_{l}}\right)} .
\end{aligned}
$$

The equalities hold if and only if factor graphs are regular.

Proof. Using the degree formula of a vertex of $V\left(\mathbf{G}_{k} \oplus \mathbf{H}_{l}\right)$ in (1),

$$
\begin{aligned}
& \operatorname{ISI}\left(\mathbf{G}_{k} \oplus \mathbf{H}_{l}\right)=\sum_{\left(g_{k}, h_{l}\right)\left(g_{k}^{\prime} h_{l}^{\prime}\right) \in E\left(\mathbf{G}_{k} \oplus \mathbf{H}_{l}\right)} \frac{d_{\mathbf{G}_{k} \oplus \mathbf{H}_{l}}\left(g_{k}, h_{l}\right) d_{\mathbf{G}_{k} \oplus \mathbf{H}_{l}}\left(g_{k}^{\prime}, h_{l}^{\prime}\right)}{d_{\mathbf{G}_{k} \oplus \mathbf{H}_{l}}\left(g_{k}, h_{l}\right)+d_{\mathbf{G}_{k} \oplus \mathbf{H}_{l}}\left(g_{k}^{\prime}, h_{l}^{\prime}\right)} \\
& =\sum_{\left(g_{k}, h_{l}\right)\left(g_{k}^{\prime} h_{l}^{\prime}\right) \in E\left(\mathbf{G}_{k} \oplus \mathbf{H}_{l}\right)} \frac{d_{\mathbf{G}_{k} \oplus \mathbf{H}_{l}}\left(g_{k}, h_{l}\right) d_{\mathbf{G}_{k} \oplus \mathbf{H}_{l}}\left(g_{k}^{\prime}, h_{l}^{\prime}\right)}{l d_{\mathbf{G}_{k}}\left(g_{k}\right)+k d_{\mathbf{H}_{l}}\left(h_{l}\right)-2 d_{\mathbf{G}_{k}}\left(g_{k}\right) d_{\mathbf{H}_{l}}\left(h_{l}\right)+l d_{\mathbf{G}_{k}}\left(g_{k}^{\prime}\right)+k d_{\mathbf{H}_{l}}\left(h_{l}^{\prime}\right)-2 d_{\mathbf{G}_{k}}\left(g_{k}^{\prime}\right) d_{\mathbf{H}_{l}}\left(h_{l}^{\prime}\right)} \\
& \leq \frac{1}{2\left(l \delta_{\mathbf{G}_{k}}+k \delta_{\mathbf{H}_{l}}-2 \delta_{\mathbf{G}_{k}} \delta_{\mathbf{H}_{l}}\right)} \sum_{\left(g_{k}, h_{l}\right)} \sum_{\left(g_{k}^{\prime} h_{l}^{\prime}\right) \in E\left(\mathbf{G}_{k} \oplus \mathbf{H}_{l}\right)} d_{\mathbf{G}_{k} \oplus \mathbf{H}_{l}}\left(g_{k}, h_{l}\right) d_{\mathbf{G}_{k} \oplus \mathbf{H}_{l}}\left(g_{k}^{\prime}, h_{l}^{\prime}\right) \\
& =\frac{M_{2}\left(\mathbf{G}_{k} \oplus \mathbf{H}_{l}\right)}{2\left(l \delta_{\mathbf{G}_{k}}+k \delta_{\mathbf{H}_{l}}-2 \delta_{\mathbf{G}_{k}} \delta_{\mathbf{H}_{l}}\right)}
\end{aligned}
$$

Similarly,

$$
\operatorname{ISI}\left(\mathbf{G}_{k} \oplus \mathbf{H}_{l}\right) \geq \frac{M_{2}\left(\mathbf{G}_{k} \oplus \mathbf{H}_{l}\right)}{2\left(l \Delta_{\mathbf{G}_{k}}+k \Delta_{\mathbf{H}_{l}}-2 \Delta_{\mathbf{G}_{k}} \Delta_{\mathbf{H}_{l}}\right)} .
$$

The above equalities hold if and only if both graphs are regular.
Next, we evaluate the bounds of inverse sum indeg (ISI) index of join of $n$ graphs.

Theorem 7. Let $\mathbf{G}_{k}=\mathbf{G}_{k_{1}}+\mathbf{G}_{k_{2}}+\cdots+\mathbf{G}_{k_{n}}$. Then,

$$
\begin{aligned}
& \sum_{s=1}^{n} \frac{M_{2}\left(\mathbf{G}_{k_{s}}\right)+\left(r-k_{s}\right) M_{1}\left(\mathbf{G}_{k_{s}}\right)+k_{s}^{\prime}\left(r-k_{s}\right)^{2}}{2\left(\Delta_{\mathbf{G}_{k_{s}}}+r-k_{s}\right)}+\frac{1}{2} \sum_{s \neq j, s, j=1}^{n} \frac{\left(2 k_{s}^{\prime}+k_{s}\left(r-k_{s}\right)\right)\left(2 k_{j}^{\prime}+k_{j}\left(r-k_{j}\right)\right)}{\Delta_{\mathbf{G}_{k_{s}}}+\Delta_{\mathbf{G}_{k_{j}}}+2 r-k_{s}-k_{j}} \\
& \leq \operatorname{ISI}\left(\mathbf{G}_{k}\right) \leq \sum_{s=1}^{n} \frac{M_{2}\left(\mathbf{G}_{k_{s}}\right)+\left(r-k_{s}\right) M_{1}\left(\mathbf{G}_{k_{s}}\right)+k_{s}^{\prime}\left(r-k_{s}\right)^{2}}{2\left(\delta_{\mathbf{G}_{k_{s}}}+r-k_{s}\right)} \\
& \quad+\frac{1}{2} \sum_{i \neq j, s, j=1}^{n} \frac{\left(2 k_{s}^{\prime}+k_{s}\left(r-k_{s}\right)\right)\left(2 k_{j}^{\prime}+k_{j}\left(r-k_{j}\right)\right)}{\delta_{\mathbf{G}_{k_{s}}}+\delta_{\mathbf{G}_{k_{j}}}+2 r-k_{s}-k_{j}} .
\end{aligned}
$$


The equalities hold if and only if $\mathbf{G}_{k_{s}}$, for $s=1,2, \ldots, n$, are regular graphs.
Proof. We assume that $\left|V\left(\mathbf{G}_{k_{s}}\right)\right|=k_{s},\left|E\left(\mathbf{G}_{k_{s}}\right)\right| \mid=k_{s}^{\prime}$ for $s=$ $1,2, \ldots, n$ and $r=k_{1}+k_{2}+\cdots+k_{n}$. By using the degree formula of a vertex in $\mathbf{G}_{k}$ given in (1),

$$
\begin{aligned}
& \operatorname{ISI}\left(\mathbf{G}_{k}\right)=\sum_{g_{k} g_{k}^{\prime} \in E\left(\mathbf{G}_{k}\right)} \frac{d_{\mathbf{G}_{k}}\left(g_{k}\right) d_{\mathbf{G}_{k}}\left(g_{k}^{\prime}\right)}{d_{\mathbf{G}_{k}}\left(g_{k}\right)+d_{\mathbf{G}_{k}}\left(g_{k}^{\prime}\right)} \\
& =\sum_{s=1}^{n} \sum_{g_{k} g_{k}^{\prime} \in E\left(\mathbf{G}_{k_{s}}\right)} \frac{\left(d_{\mathbf{G}_{k_{s}}}\left(g_{k}\right)+r-k_{s}\right)\left(d_{\mathbf{G}_{k_{s}}}\left(g_{k}^{\prime}\right)+r-k_{s}\right)}{d_{\mathbf{G}_{k_{s}}}\left(g_{k}\right)+d_{\mathbf{G}_{k_{s}}}\left(g_{k}^{\prime}\right)+2 r-2 k_{s}}+\frac{1}{2} \sum_{\substack{s \neq j, j \\
s, j=1}}^{n} \sum_{g_{k} \in V\left(\mathbf{G}_{k_{s}}\right)} \sum_{g_{k}^{\prime} \in V\left(\mathbf{G}_{k_{j}}\right)} \frac{\left(d_{\mathbf{G}_{k_{s}}}\left(g_{k}\right)+r-k_{s}\right)-\left(d_{\mathbf{G}_{k_{j}}}\left(g_{k}^{\prime}\right)+r-k_{j}\right)}{d_{\mathbf{G}_{k_{s}}}\left(g_{k}\right)+d_{\mathbf{G}_{k_{j}}}\left(g_{k}^{\prime}\right)+2 r-k_{s}-k_{j}} \\
& \leq \sum_{s=1}^{n} \sum_{g_{k} g_{k}^{\prime} \in E\left(\mathbf{G}_{k_{s}}\right)} \frac{\left(d_{\mathbf{G}_{k_{s}}}\left(g_{k}\right)+r-k_{s}\right)\left(d_{\mathbf{G}_{k_{s}}}\left(g_{k}^{\prime}\right)+r-k_{s}\right)}{\delta_{\mathbf{G}_{k_{s}}}+\delta_{\mathbf{G}_{k_{s}}}+2 r-2 k_{s}}+\frac{1}{2} \sum_{\substack{s \neq j, j \\
s, j=1}}^{n} \sum_{g_{k} \in V\left(\mathbf{G}_{k_{s}}\right)} \sum_{v \in V\left(\mathbf{G}_{k_{j}}\right)} \frac{\left(d_{\mathbf{G}_{k_{s}}}\left(g_{k}\right)+r-k_{s}\right)\left(d_{\mathbf{G}_{k_{j}}}\left(g_{k}^{\prime}\right)+r-k_{j}\right)}{\delta_{\mathbf{G}_{k_{s}}}+\delta_{\mathbf{G}_{k_{j}}}+2 r-k_{s}-k_{j}} \\
& =\sum_{s=1}^{n} \frac{M_{2}\left(\mathbf{G}_{k_{s}}\right)+\left(r-k_{s}\right) M_{1}\left(\mathbf{G}_{k_{s}}\right)+k_{s}^{\prime}\left(r-k_{s}\right)^{2}}{2\left(\delta_{\mathbf{G}_{k_{s}}}+r-k_{s}\right)}+\frac{1}{2} \sum_{\substack{s \neq j, s, j=1}}^{n} \frac{\left(2 k_{s}^{\prime}+k_{s}\left(r-k_{s}\right)\right)\left(2 k_{j}^{\prime}+k_{j}\left(r-k_{j}\right)\right)}{\delta_{\mathbf{G}_{k_{s}}}+\delta_{\mathbf{G}_{k_{j}}}+2 r-k_{s}-k_{j}} .
\end{aligned}
$$

Similarly

$$
\operatorname{ISI}\left(\mathbf{G}_{k}\right) \geq \sum_{s=1}^{n} \frac{M_{2}\left(\mathbf{G}_{k_{s}}\right)+\left(r-k_{s}\right) M_{1}\left(\mathbf{G}_{k_{s}}\right)+k_{s}^{\prime}\left(r-k_{s}\right)^{2}}{2\left(\Delta_{\mathbf{G}_{k_{s}}}+r-k_{s}\right)}+\frac{1}{2} \sum_{\substack{s \neq j, s, j=1}}^{n} \frac{\left(2 k_{s}^{\prime}+k_{s}\left(r-k_{s}\right)\right)\left(2 k_{j}^{\prime}+k_{j}\left(r-k_{j}\right)\right)}{\Delta_{\mathbf{G}_{k_{s}}}+\Delta_{\mathbf{G}_{k_{j}}}+2 r-k_{s}-k_{j}} .
$$

The above equalities hold if and only if $\mathbf{G}_{k_{s}}$, $s=1,2, \ldots, n$, are regular.

In the following theorem, we calculate the bounds for ISI index of $\mathbf{G}_{k} \circ \mathbf{H}_{l}$.
Theorem 8. Let $\mathbf{G}_{k}$ and $\mathbf{H}_{l}$ be k-vertex and l-vertex graphs. Then,

$$
\begin{aligned}
& \frac{k\left(M_{2}\left(\mathbf{H}_{l}\right)+M_{1}\left(\mathbf{H}_{l}\right)+l\right)}{2\left(\Delta_{\mathbf{H}_{l}}+1\right)}+\frac{\left(2 l^{\prime}+l\right)\left(2 k^{\prime}+k l\right)}{\Delta_{\mathbf{G}_{k}}+\Delta_{\mathbf{H}_{l}}+l+1}+\frac{M_{2}\left(\mathbf{G}_{k}\right)+l M_{1}\left(\mathbf{G}_{k}\right)+l^{2} l^{\prime}}{2\left(\Delta_{\mathbf{G}_{k}}+l\right)} \leq \operatorname{ISI}\left(\mathbf{G}_{k} \circ \mathbf{H}_{l}\right) \\
& \leq \frac{k\left(M_{2}\left(\mathbf{H}_{l}\right)+M_{1}\left(\mathbf{H}_{l}\right)+l\right)}{2\left(\delta_{\mathbf{H}_{l}}+1\right)}+\frac{\left(2 l^{\prime}+l\right)\left(2 k^{\prime}+k l\right)}{\delta_{\mathbf{G}_{k}}+\delta_{\mathbf{H}_{l}}+l+1}+\frac{M_{2}\left(\mathbf{G}_{k}\right)+l M_{1}\left(\mathbf{G}_{k}\right)+l^{2} l^{\prime}}{2\left(\delta_{\mathbf{G}_{k}}+l\right)} .
\end{aligned}
$$


The equalities hold if and only if both graphs are regular.

Proof. Using the degree formula of a vertex in corona product in (1),

$$
\begin{aligned}
\operatorname{ISI}\left(\mathbf{G}_{k} \circ \mathbf{H}_{l}\right)= & k \sum_{h_{l} h_{l}^{\prime} \in E\left(\mathbf{H}_{l}\right)} \frac{\left(d_{\mathbf{H}_{l}}\left(h_{l}\right)+1\right)\left(d_{\mathbf{H}_{l}}\left(h_{l}^{\prime}\right)+1\right)}{d_{\mathbf{H}_{l}}\left(h_{l}\right)+d_{\mathbf{H}_{l}}\left(h_{l}^{\prime}\right)+2}+\sum_{s=1}^{k} \sum_{j=1}^{l} \frac{\left(d_{\mathbf{H}_{l}}\left(h_{l_{j}}\right)+1\right)\left(d_{\mathbf{G}_{k}}\left(g_{k_{s}}\right)+l\right)}{d_{\mathbf{H}_{l}}\left(h_{l_{j}}\right)+d_{\mathbf{G}_{k}}\left(g_{k_{s}}\right)+l+1} \\
& +\sum_{g_{k} g_{k}^{\prime} \in E\left(\mathbf{G}_{k}\right)} \frac{\left(d_{\mathbf{G}_{k}}\left(g_{k}\right)+l\right)\left(d_{\mathbf{G}_{k}}\left(g_{k}^{\prime}\right)+l\right)}{d_{\mathbf{G}_{k}}\left(g_{k}\right)+d_{\mathbf{G}_{k}}\left(g_{k}^{\prime}\right)+2 l} .
\end{aligned}
$$

From equation (2), we obtain

$$
\begin{aligned}
\operatorname{ISI}\left(\mathbf{G}_{k} \circ \mathbf{H}_{l}\right) \leq & k \sum_{h_{l} h_{k} \in E\left(\mathbf{H}_{l}\right)} \frac{\left(d_{\mathbf{H}_{l}}\left(h_{l}\right)+1\right)\left(d_{\mathbf{H}_{l}}\left(h_{l}^{\prime}\right)+1\right)}{2\left(\delta_{\mathbf{H}_{l}}+1\right)}+\sum_{s=1}^{k} \sum_{j=1}^{l} \frac{\left(d_{\mathbf{H}_{l}}\left(h_{l_{j}}\right)+1\right)\left(d_{\mathbf{G}_{k}}\left(g_{k_{s}}\right)+l\right)}{\delta_{\mathbf{G}_{k}}+\delta_{\mathbf{H}_{l}}+l+1} \\
& +\sum_{g_{k} g_{k}^{\prime} \in E\left(\mathbf{G}_{k}\right)} \frac{\left(d_{\mathbf{G}_{k}}\left(g_{k}\right)+l\right)\left(d_{\mathbf{G}_{k}}\left(g_{k}^{\prime}\right)+l\right)}{2\left(\delta_{\mathbf{G}_{k}}+l\right)} \\
= & \frac{k\left(M_{2}\left(\mathbf{H}_{l}\right)+M_{1}\left(\mathbf{H}_{l}\right)+l\right)}{2\left(\delta_{\mathbf{H}_{l}}+1\right)}+\frac{\left(2 l^{\prime}+l\right)\left(2 k^{\prime}+k l\right)}{\delta_{\mathbf{G}_{k}}+\delta_{\mathbf{H}_{l}}+l+1}+\frac{M_{2}\left(\mathbf{G}_{k}\right)+l k \prime}{2\left(\mathbf{G}_{k}\right)+l^{2} l^{\prime}} \\
&
\end{aligned}
$$

Similarly, we calculate

$$
\operatorname{ISI}\left(\mathbf{G}_{k} \circ \mathbf{H}_{l}\right) \geq \frac{k\left(M_{2}\left(\mathbf{H}_{l}\right)+M_{1}\left(\mathbf{H}_{l}\right)+l\right)}{2\left(\Delta_{\mathbf{H}_{l}}+1\right)}+\frac{\left(2 l^{\prime}+l\right)\left(2 k^{\prime}+k l\right)}{\Delta_{\mathbf{G}_{k}}+\Delta_{\mathbf{H}_{l}}+l+1}+\frac{M_{2}\left(\mathbf{G}_{k}\right)+l M_{1}\left(\mathbf{G}_{k}\right)+l^{2} l^{\prime}}{2\left(\Delta_{\mathbf{G}_{k}}+l\right)} .
$$

The above equalities hold only when $\mathbf{G}_{k}$ and $\mathbf{H}_{l}$ are regular graphs.
Next, we evaluate the bounds for inverse sum indeg (ISI) index of Indu-Bala product. 
Theorem 9. Let $\mathbf{G}_{k}$ and $\mathbf{H}_{l}$ be k-vertex and l-vertex graphs.

Then,

$$
\begin{aligned}
\frac{M_{2}\left(\mathbf{G}_{k}\right)+l M_{1}\left(\mathbf{G}_{k}\right)+l^{2} k^{\prime}}{\Delta_{\mathbf{G}_{k}}+l}+ & \frac{2 M_{2}\left(\mathbf{H}_{l}\right)+(2 l+3) M_{1}\left(\mathbf{H}_{l}\right)+\left(2 l^{\prime}+l\right)(k+1)^{2}+4 l^{\prime}(k+1)}{2\left(\Delta_{\mathbf{H}_{l}}+k+1\right)} \\
& +\frac{2\left(4 k^{\prime} l^{\prime}+2 k^{\prime} l(k+1)+2 l^{\prime} k l+l^{2} k(k+1)\right)}{\Delta_{\mathbf{G}_{k}}+\Delta_{\mathbf{H}_{l}}+k+l+1} \leq \operatorname{ISI}\left(\mathbf{G}_{k} \nabla \mathbf{H}_{l}\right) \leq \frac{M_{2}\left(\mathbf{G}_{k}\right)+l M_{1}\left(\mathbf{G}_{k}\right)+l^{2} k^{\prime}}{\delta_{\mathbf{G}_{k}}+l} \\
& +\frac{2 M_{2}\left(\mathbf{H}_{l}\right)+(2 l+3) M_{1}\left(\mathbf{H}_{l}\right)+\left(2 l^{\prime}+l\right)(k+1)^{2}+4 l^{\prime}(k+1)}{2\left(\delta_{\mathbf{H}_{l}}+k+1\right)} \\
& +\frac{2\left(4 k^{\prime} l^{\prime}+2 k^{\prime} l(k+1)+2 l^{\prime} k l+l^{2} k(k+1)\right)}{\delta_{\mathbf{G}_{k}}+\delta_{\mathbf{H}_{l}}+k+l+1} .
\end{aligned}
$$

The equalities hold only when $\mathbf{G}_{k}$ and $\mathbf{H}_{l}$ are regular.

Proof. Using the degree formula of a vertex in Indu-Bala product in (1),

$$
\begin{aligned}
\operatorname{ISI}\left(\mathbf{G}_{k} \nabla \mathbf{H}_{l}\right)= & 2\left[\sum_{g_{k} g_{k}^{\prime} \in E\left(\mathbf{G}_{k}\right)} \frac{\left(d_{\mathbf{G}_{k}}\left(g_{k}\right)+l\right)\left(d_{\mathbf{G}_{k}}\left(g_{k}^{\prime}\right)+l\right)}{\left(d_{\mathbf{G}_{k}}\left(g_{k}\right)+d_{\mathbf{G}_{k}}\left(g_{k}^{\prime}\right)\right)+2 l}+\sum_{h_{l} h_{k}^{\prime} \in E\left(\mathbf{H}_{l}\right)} \frac{\left(d_{\mathbf{H}_{l}}\left(h_{l}\right)+k+1\right)\left(d_{\mathbf{H}_{l}}\left(h_{l}^{\prime}\right)+k+1\right)}{d_{\mathbf{H}_{l}}\left(h_{l}\right)+d_{\mathbf{H}_{l}}\left(h_{l}^{\prime}\right)+2 k+2}\right. \\
& \left.+\sum_{g_{k} \in V\left(\mathbf{G}_{k}\right)} \sum_{h_{l} \in V\left(\mathbf{H}_{l}\right)} \frac{\left(d_{\mathbf{G}_{k}}\left(g_{k}\right)+l\right)\left(d_{\mathbf{H}_{l}}\left(h_{l}\right)+k+1\right)}{d_{\mathbf{G}_{k}}\left(g_{k}\right)+d_{\mathbf{H}_{l}}\left(h_{l}\right)+k+l+1}\right]+\sum_{h_{l} \in V\left(\mathbf{H}_{l}\right)} \frac{\left(d_{\mathbf{H}_{l}}\left(h_{l}\right)+k+1\right)^{2}}{2\left(d_{\mathbf{H}_{l}}\left(h_{l}\right)+k+1\right)} .
\end{aligned}
$$

Using equation (2), then we have

$$
\begin{aligned}
\operatorname{ISI}\left(\mathbf{G}_{k} \boldsymbol{\nabla} \mathbf{H}_{l}\right) \leq 2\left[\sum_{g_{k} g_{k}^{\prime} \in E\left(\mathbf{G}_{k}\right)} \frac{\left(d_{\mathbf{G}_{k}}\left(g_{k}\right)+l\right)\left(d_{\mathbf{G}_{k}}\left(g_{k}^{\prime}\right)+l\right)}{2\left(\delta_{\mathbf{G}_{k}}+l\right)}+\sum_{h_{l} h^{\prime} \in E\left(\mathbf{H}_{l}\right)} \frac{\left(d_{\mathbf{H}_{l}}\left(h_{l}\right)+k+1\right)\left(d_{\mathbf{H}_{l}}\left(h_{l}^{\prime}\right)+k+1\right)}{2\left(\delta_{\mathbf{H}_{l}}+k+1\right)}\right. \\
\left.+\sum_{g_{k} \in V\left(\mathbf{G}_{k}\right)} \sum_{h_{l} \in V\left(\mathbf{H}_{l}\right)} \frac{\left(d_{\mathbf{G}_{k}}\left(g_{k}\right)+l\right)\left(d_{\mathbf{H}_{l}}\left(h_{l}\right)+k+1\right)}{\delta_{\mathbf{G}_{k}}+\delta_{\mathbf{H}_{l}}+k+l+1}\right]+\sum_{h_{l} \in V\left(\mathbf{H}_{l}\right)} \frac{\left(d_{\mathbf{H}_{l}}\left(h_{l}\right)+k+1\right)^{2}}{2\left(\delta_{\mathbf{H}_{l}}+k+1\right)} \\
=\frac{M_{2}\left(\mathbf{G}_{k}\right)+l M_{1}\left(\mathbf{G}_{k}\right)+l^{2} k^{\prime}}{\delta_{\mathbf{G}_{k}}+l}+\frac{2 M_{2}\left(\mathbf{H}_{l}\right)+(2 l+3) M_{1}\left(\mathbf{H}_{l}\right)+\left(2 l^{\prime}+l\right)(k+1)^{2}+4 l^{\prime}(k+1)}{2\left(\delta_{\mathbf{H}_{l}}+k+1\right)} \\
+\frac{2\left(4 k^{\prime} l^{\prime}+2 k^{\prime} l(k+1)+2 l^{\prime} k l+l^{2} k(k+1)\right)}{\delta_{\mathbf{G}_{k}}+\delta_{\mathbf{H}_{l}}+k+l+1} .
\end{aligned}
$$

Similarly, we calculate 


$$
\begin{aligned}
\operatorname{ISI}\left(\mathbf{G}_{k} \boldsymbol{\nabla} \mathbf{H}_{l}\right) \geq & \frac{M_{2}\left(\mathbf{G}_{k}\right)+l M_{1}\left(\mathbf{G}_{k}\right)+l^{2} k^{\prime}}{\Delta_{\mathbf{G}_{k}}+l}+\frac{2 M_{2}\left(\mathbf{H}_{l}\right)+(2 l+3) M_{1}\left(\mathbf{H}_{l}\right)+\left(2 l^{\prime}+l\right)(k+1)^{2}+4 l^{\prime}(k+1)}{2\left(\Delta_{\mathbf{H}_{l}}+k+1\right)} \\
& +\frac{2\left(4 k^{\prime} l l+2 k^{\prime} l(k+1)+2 l^{\prime} k l+l^{2} k(k+1)\right)}{\Delta_{\mathbf{G}_{k}}+\Delta_{\mathbf{H}_{l}}+k+l+1} .
\end{aligned}
$$
graphs.

The equalities hold only when $\mathbf{G}_{k}$ and $\mathbf{H}_{l}$ are regular

In the next theorem, we find the inverse sum indeg (ISI) index of double graph.

Theorem 10. Let $\mathbf{G}_{k}$ be a k-vertex graph. Then,

$$
\operatorname{ISI}\left(D\left[\mathbf{G}_{k}\right]\right)=8 \operatorname{ISI}\left(\mathbf{G}_{k}\right) .
$$

Proof. Using the degree formula of a vertex in $D\left[\mathbf{G}_{k}\right]$ in equation (1), we acquire

$$
\begin{aligned}
\operatorname{ISI}\left(D\left[\mathbf{G}_{k}\right]\right) & =\sum_{g_{k} g_{k}^{\prime} \in E\left(D\left[\mathbf{G}_{k}\right]\right)} \frac{d_{D\left[\mathbf{G}_{k}\right]}\left(g_{k}\right) d_{D\left[\mathbf{G}_{k}\right]}\left(g_{k}^{\prime}\right)}{d_{D\left[\mathbf{G}_{k}\right]}\left(g_{k}\right)+d_{D\left[\mathbf{G}_{k}\right]}\left(g_{k}^{\prime}\right)} \\
& =4 \sum_{g_{k} g_{k}^{\prime} \in E\left(\mathbf{G}_{k}\right)} \frac{\left(2 d_{\mathbf{G}_{k}}\left(g_{k}\right)\right)\left(2 d_{\mathbf{G}_{k}}\left(g_{k}^{\prime}\right)\right)}{2 d_{\mathbf{G}_{k}}\left(g_{k}\right)+2 d_{\mathbf{G}_{k}}\left(g_{k}^{\prime}\right)} \\
& =8 \sum_{g_{k} g_{k}^{\prime} \in E\left(\mathbf{G}_{k}\right)} \frac{d_{\mathbf{G}_{k}}\left(g_{k}\right) d_{\mathbf{G}_{k}}\left(g_{k}^{\prime}\right)}{d_{\mathbf{G}_{k}}\left(g_{k}\right)+d_{\mathbf{G}_{k}}\left(g_{k}^{\prime}\right)}=8 \operatorname{ISI}\left(\mathbf{G}_{k}\right) .
\end{aligned}
$$

In the upcoming theorem, we calculate the bounds for inverse sum indeg (ISI) index of strong double graph.

Theorem 11. Let $\mathbf{G}_{k}$ be an k-vertex graph. Then,

$$
\frac{M_{2}\left(\operatorname{SD}\left[\mathbf{G}_{k}\right]\right)}{2\left(2 \Delta_{\mathbf{G}_{k}}+1\right)} \leq \operatorname{ISI}\left(\operatorname{SD}\left[\mathbf{G}_{k}\right]\right) \leq \frac{M_{2}\left(\operatorname{SD}\left[\mathbf{G}_{k}\right]\right)}{2\left(2 \delta_{\mathbf{G}_{k}}+1\right)}
$$

The equalities hold only when $\mathbf{G}_{k}$ is regular.

Proof. Using the degree formula of a vertex in $\mathrm{SD}\left[\mathbf{G}_{k}\right]$ in (1),

$$
\begin{aligned}
\operatorname{ISI}\left(\operatorname{SD}\left[\mathbf{G}_{k}\right]\right) & =\sum_{g_{k} g_{k}^{\prime} \in E\left(\operatorname{SD}\left[\mathbf{G}_{k}\right]\right)} \frac{d_{\left(\mathrm{SD}\left[\mathbf{G}_{k}\right]\right)}\left(g_{k}\right) d_{\left(\mathrm{SD}\left[\mathbf{G}_{k}\right]\right)}\left(g_{k}^{\prime}\right)}{d_{\left(\mathrm{SD}\left[\mathbf{G}_{k}\right]\right)}\left(g_{k}\right)+d_{\left(\mathrm{SD}\left[\mathbf{G}_{k}\right]\right)}\left(g_{k}^{\prime}\right)} \\
& =\sum_{g_{k} g_{k}^{\prime} \in E\left(\operatorname{SD}\left[\mathbf{G}_{k}\right]\right)} \frac{d_{\left(\mathrm{SD}\left[\mathbf{G}_{k}\right]\right)}\left(g_{k}\right) d_{\left(\mathrm{SD}\left[\mathbf{G}_{k}\right]\right)}\left(g_{k}^{\prime}\right)}{2 d_{\mathbf{G}_{k}}\left(g_{k}\right)+1+2 d_{\mathbf{G}_{k}}\left(g_{k}^{\prime}\right)+1} \\
& \leq \sum_{u v \in E\left(\operatorname{SD}\left[\mathbf{G}_{k}\right]\right)} \frac{d\left(\operatorname{SD}\left[\mathbf{G}_{k}\right]\right)}{2\left(g_{k}\right) d\left(\operatorname{SD}\left[\mathbf{G}_{k}\right]\right)}\left(g_{k}^{\prime}\right) \\
& =\frac{M_{2}\left(\operatorname{SD}\left[\mathbf{G}_{k}\right]\right)}{2\left(2 \delta_{\mathbf{G}_{k}}+1\right)} .
\end{aligned}
$$

Similarly, we compute

$$
\operatorname{ISI}\left(\operatorname{SD}\left[\mathbf{G}_{k}\right]\right) \geq \frac{M_{2}\left(\operatorname{SD}\left[\mathbf{G}_{k}\right]\right)}{2\left(2 \Delta_{\mathbf{G}_{k}}+1\right)} \text {. }
$$

The above equalities hold only when $\mathbf{G}_{k}$ is a regular graph.

\section{Conclusion}

In this paper, some graph operations including different products, differences, union of graphs, double graph, and strong double graph are studied. In particular, we have found the sharp bounds for inverse sum indeg (ISI) index of these operations of graphs. The investigation related to other significant predictors is still open.

\section{Data Availability}

All kinds of data and materials, used to compute the results, are provided in Section 1.

\section{Conflicts of Interest}

The authors declare that they have no conflicts of interest. 


\section{Acknowledgments}

This project was sponsored by the Deanship of Scientific Research under Nasher Proposal No. 216006, King Faisal University.

\section{References}

[1] D. Vuki, ević and M. Nazlić, "Bond aditive mdelling 1. Ariatic indices," Croatica Chemica Acta, vol. 83, pp. 243-260, 2010.

[2] J. Sedlar, D. Stevanović, and A. Vasilyev, "On the inverse sum indeg index," Discrete Applied Mathematics, vol. 184, pp. 202-212, 2015.

[3] F. Falahati-Nezhad, M. Azari, and T. Došlić, "Sharp bounds on the inverse sum indeg index," Discrete Applied Mathematics, vol. 217, pp. 185-195, 2017.

[4] I. Gutman and N. Trinajstić, "Graph theory and molecular orbitals. Total $\varphi$-electron energy of alternant hydrocarbons $\pi$ electron energy of alternant hydro-carbons," Chemical Physics Letters, vol. 17, no. 4, pp. 535-538, 1972.

[5] A. R. Ashrafi, M. H. Khalifeh, and H. Yousefi-Azari, "The first and second zagreb indices of some graphs operations," Discrete Applied Mathematics, vol. 157, pp. 804-811, 2009.

[6] A. R. Ashrafi and Z. Yarahmadi, "The szeged, vertex PI, first and second zagreb indices of corona product of graphs," Filomat, vol. 3, no. 3, pp. 467-472, 2012.

[7] A. R. Ashrafi, T. Došlić, and A. Hamzeh, "The zagreb coindices of graph operations," Discrete Applied Mathematics, vol. 158, no. 15, pp. 1571-1578, 2010.

[8] M. Aaari and A. Iranmanesh, "Some inequalities for the multiplicative sum zagreb index of graph operations," Journal of Mathematical Inequalities, vol. 9, pp. 727-738, 2015.

[9] B. Basavanagoud, S. Patil, V. R. Desai, and S. M. Hosamani, "Computing certain topological indices of indu-bala product of graphs," 2016.

[10] N. De, S. M. A. Nayeem, and A. Pal, "F-index of some graph operations," Discrete Mathematics, Algorithms and Applications, vol. 8, no. 2, p. 1650025, 2016.

[11] G. Indulal and R. Balakrishnan, "Distance spectrum of InduBala product of graphs," AKCE International Journal of Graphs and Combinatorics, vol. 13, no. 3, pp. 230-234, 2016.

[12] E. Munarini, A. Scagliola, C. P. Cippo, and N. Salvi, "Double graphs," Discrete Mathematics, vol. 308, no. 2-3, pp. 242-254, 2008.

[13] M. S. Marino and N. Z. Salvi, "Generalizing double graphs," Atti della Accademia Peloritana dei Pericolanti-Classe di Scienze Fisiche Matematiche e Naturali, vol. 85, no. 2, 2006.

[14] S. Pirzada and H. A. Ganie, "Spectra, energy and laplacian energy of strong double graphs," Mathematical Technology of Networks, vol. 128, pp. 175-189, 2015.

[15] S. Akhter, Z. Iqbal, A. Aslam, and W. Gao, "Mostar index of graph operations," 2020, https://arxiv.org/abs/2005.09416.

[16] W. Gao, Z. Iqbal, Z. Iqbal, S. Akhter, M. Ishaq, and A. Aslam, "On irregularity descriptors of derived graphs," AIMS Mathematics, vol. 5, no. 5, pp. 4085-4107, 2020.

[17] M. Imran, S. Akhter, and Z. Iqbal, "Edge Mostar index of chemical structures and nanostructures using graph operations," Int. J. Quantum. Chem., vol. 120, no. 15, Article ID e26259, 2020.

[18] M. Imran, S. Akhter, and Z. Iqbal, "On the eccentric connectivity polynomial of F-sum of connected graphs," Complexity, vol. 2020, Article ID 5061682, 9 pages, 2020.

[19] H. Yang, M. Imran, S. Akhter, Z. Iqbal, and M. K. Siddiqui, "On distance-based topological descriptors of subdivision vertex-edge join of three graphs," IEEE Access, vol. 7, pp. 143381-143391, 2019.

[20] S. Akhter and M. Imran, "The sharp bounds on general sumconnectivity index of four operations on graphs," Journal of Inequalities and Applications, vol. 2016, no. 1, pp. 241-250, 2016.

[21] S. Akhter, M. Imran, and Z. Raza, "On the general sumconnectivity index and general randić index of cacti," Journal of Inequalities and Applications, vol. 2016, no. 1, pp. 300-309, 2016.

[22] S. Akhter and M. Imran, "Computing the forgotten topological index of four operations on graphs," AKCE International Journal of Graphs and Combinatorics, vol. 14, no. 1, pp. 70-79, 2017.

[23] M. Imran, S. Baby, H. M. A. Siddiqui, and M. K. Shafiq, "On the bounds of degree-based topological indices of the cartesian product of F-sum of connected graphs," Journal of Inequalities and Applications, vol. 2017, no. 1, pp. 305-315, 2017.

[24] H. M. A. Siddiqui, S. Baby, and M. K. Shafiq, "The sharp bounds of topological indices of strong product of new F-sum on connected graphs," Utilitas Mathematica, vol. 112, pp. 139-156, 2017.

[25] Z. Yarahmadi, "Computing some topological indices of tensor product of graphs," Iranian Journal of Mathematical Chemistry, vol. 2, no. 1, pp. 109-118, 2011. 